\title{
Breakup Length of Electrified Liquid Jets: Scaling Laws and Applications
}

\author{
A. Said Ismail, ${ }^{*}$ J. Yao, ${ }^{\dagger}$ H.H. Xia, and J.P.W. Stark \\ School of Engineering and Materials Science, Queen Mary University of London, London E1 4NS, \\ United Kingdom
}

(Received 2 July 2018; revised manuscript received 5 October 2018; published XX XX 2018)

\begin{abstract}
The growth rate of infinitesimal perturbations in electrified jets exhibits different behavior at the viscid and inviscid limits. Using Saville's approach to estimate the growth rate for perturbations in the longwavelength limit and by ignoring the effects of gravity, we derive two scaling laws for the jet breakup length in two regimes of the Taylor-cone mode. Our experimental measurements show clear dependency of the jet length on the flow rate; however, change of the applied voltage appears to affect only the cone angle but not the jet itself. The experimental data agree excellently with our theoretical model in both cases. On the basis of the jet diameter, the transition between viscid and inviscid limits appears to occur at an electric Reynolds number of approximately 5. Finally, we show how to enhance the quality and the resolution in electrostatic-inkjet-printing applications by setting the printing distance less than the jet length and predict the line width as a function of the operational parameters.
\end{abstract}

DOI: 10.1103/PhysRevApplied.0.XXXXXX

\section{INTRODUCTION}

The breakup of liquid jets in the capillary regime, which is caused by the growth of perturbations, has been a focus for many scientists over several decades. The first attempt to explain the capillary instability was undertaken more than 100 years ago by Rayleigh [1]. He showed that axisymmetric disturbances grow downstream until a breakup happens for uncharged inviscid liquid jets if the wavelength exceeds the circumference of the jet. For gravitational jets, Javadi et al. [2] introduced a theory supported by experiments that explains how viscosity tends to increase the jet intact length. If the jet is produced out of a liquid meniscus as a result of application of an electric field, the so-called cone-jet mode or Taylor-cone mode, the instability mechanism will be different. Local stability analysis has been used extensively to understand the breakup mechanism of electrified jets since the analysis of Taylor [3]. Taylor showed that the electric charge stabilizes the jet for long-wavelength perturbations, correcting errors in Basset's analysis [4]. Saville [5] adopted a linear theory to estimate the growth rate of both axisymmetric and nonaxisymmetric perturbations in electrified jets taking into account the effect of viscosity and inertia. There have also been studies related to the nonlinear breakup of an electrified jet, but in which details of the

\footnotetext{
*a.ismail@qmul.ac.uk

†J.Yao@qmul.ac.uk
}

breakup process cannot be captured with simplified onedimensional models. As an example, Conroy et al. [6] used a nonlinear slender jet model to investigate ionic surfactants and electrokinetic influences on the size of satellite droplets produced at jet breakup; however, their numerical simulations suggest that the effect on the breakup time is weak. In contrast, Collins et al. [7] solved the full Navier-Stokes equations assuming a perfectly conducting cylindrical jet, which is bounded by a concentric ground electrode, and they showed that nonlinear effects tend to delay the breakup process, and hence increase jet length. The configuration investigated by Collins et al. is, however, different from that of investigated by Saville [5], who assumed a ground electrode at infinity. Knowing the length of electrified jets is crucial in many fields, such as analytical chemistry, fabrication of nanofibers and biological materials, the textile industry, and continuous inkjet printing. Although much research has been conducted into the breakup of electrified jets for inviscid liquids [8-10] and highly viscous liquids [7,11-13], none of these have introduced a quantitative estimation for the breakup length. In this work, we propose two scaling laws to predict the breakup length of an electrified jet in the viscid and inviscid limits on the basis of Saville's approach to estimate the growth rate of perturbations in the long-wavelength limit. These scaling laws are set in the context of a systematic experimental investigation. We then use these scaling laws to print grids from nanoparticle inks with high quality and high resolution, giving an accurate prediction of the width of the printed lines. 


\section{SCALING OF THE JET LENGTH IN THE VISCID LIMIT}

If we consider a liquid meniscus emanating from a metal nozzle at a voltage difference $V$ that is applied between the nozzle and a counter-electrode plate, electric charges will accumulate on the liquid surface, producing stresses that deform the meniscus into a cone shape. Because of the singularity created at the apex, a fine jet, much smaller than the nozzle, can be produced from the cone. At relatively low voltage, a pulsating mode occurs in which the ejection is periodic. If the voltage is increased above a critical value, a steady jet will be emitted, which then breaks up at some point downstream (Taylor-cone mode). The jet produced in this mode typically breaks up into either an axisymmetric fashion (varicose) or a nonaxisymmetric fashion (whipping). Our focus in this study is on the intact length of the jet in the Taylor-cone mode when the breakup is axisymmetric. The parameters that mainly characterize the problem are the density $\rho$, dynamic viscosity $\mu$, surface tension $\gamma$, electrical conductivity $K$, electrical permittivity of the environment $\varepsilon_{0}$, relative electrical permittivity $\beta$, flow rate $Q$, and jet radius $R$. Here the jet diameter is assumed to be constant as the thinning of the jet is negligible. If we consider an approximation of a linear theory and use the viscous time $t_{\mu}=\mu R / \gamma$ and the viscous velocity $v_{\mu}=\gamma / \mu$ as characteristic parameters and if we ignore the effects of gravity, the nondimensional equations that govern the jet can be written as follows:

$$
\begin{gathered}
\nabla \cdot \mathbf{v}=0 \\
\frac{1}{\mathrm{Oh}^{2}} \frac{\partial}{\partial t} \mathbf{v}=-\nabla p+\nabla^{2} \mathbf{v},
\end{gathered}
$$

where the Ohnesorge number $\mathrm{Oh}=\mu / \sqrt{\rho \gamma R}$. The infinitesimal perturbations $\{\tilde{u}, \tilde{v}, \tilde{w}, \tilde{p}\}$ for the velocity and the pressure that propagate through the jet, written in cylindrical coordinates $(r, z)$, read

$$
\{\tilde{u}, \tilde{v}, \tilde{w}, \tilde{p}\}=\{u, v, w, p\}(r, z) \times \exp (\omega t),
$$

where $u, v, w$, and $p$ are the velocities and the pressure amplitude, and $\omega$ is the growth rate. Saville solved Eqs. (1)-(3) together with Maxwell's equations, assuming that the ground electrode is at infinity, to obtain a dispersion relation. Considering that the most-unstable axisymmetric perturbation corresponds to an infinitely long wavelength, he then estimated the growth rate for a viscous jet breaking up through a varicose mode [5] to be

$$
\omega \sim \frac{\gamma}{6 \mu R}\left(1-\frac{R \varepsilon_{0} E_{0}^{2}}{4 \pi \gamma}\right)
$$

where $E_{0}$ is the electric field strength at the surface of the jet. In Eq. (4), the term in parentheses has to be less than 1 for the jet to have axisymmetric breakup, which is the case for all the experimental data in this study.

We now extend this approach to identify the breakup length of the jet. To do this, Eq. (4) can be used to estimate the breakup time $t_{b}$ for the jet, where $t_{b} \sim 1 / \omega$, from which, if $v_{j}$ is the jet velocity, the breakup length of the jet is

$$
L_{b} \sim v_{j} t_{b}
$$

Knowing the jet velocity $v_{j} \sim Q / R^{2}$ from the continuity equation and the electric field strength $E_{0}$ estimated from $E_{0}=\left(\rho \gamma^{2} K^{2} / \varepsilon_{0}^{5}\right)^{1 / 6}$ [14], we see the only unknown in Eq. (5) is the jet radius. Gañán-Calvo [15] suggested a scaling law for the jet radius. This was subsequently validated experimentally in Ref. [16]:

$$
R=\left(\frac{\rho Q^{3} \varepsilon_{0}}{\gamma K}\right)^{1 / 6} .
$$

This relation is limited to the following conditions:

$$
\alpha_{\rho} \gg \alpha_{\mu}^{1 / 4}, \quad \alpha_{\rho} /(\beta-1) \gg 1,
$$

where $\alpha_{\rho}=\rho K Q / \gamma \varepsilon_{0}$ and $\alpha_{\mu}=K^{2} \mu^{3} Q / \varepsilon_{0}^{2} \gamma^{3}$. These conditions hold for the experimental scope considered in this study.

From Eqs. (4)-(6), the scaling for the jet breakup length can be written as

$$
L_{b}=k_{v} \frac{6 \mu}{Z_{v}},
$$

where $k_{v}$ is a constant and

$$
Z_{v}=\left[\left(\frac{\rho \gamma^{5} \varepsilon_{0}}{Q^{3} K}\right)^{1 / 6}-\frac{1}{4 \pi}\left(\frac{\rho^{2} \gamma K}{\varepsilon_{0}}\right)^{1 / 3}\right] .
$$

\section{EXPERIMENT}

To validate this scaling law, we measure the jet breakup length for different liquids with a wide range of properties. The jet length $L_{b}$ is measured from the point where the change of the cone jet diameter starts to be very small $\left(\Delta d_{j} / \Delta z<0.4\right.$, where $z$ represents the coordinate in the axial direction). The liquids used are ethanol, ethylene glycol, 2-propanol, 1-methoxy-2-propanol, propylene carbonate, triethylene glycol, dipropylene glycol, glycerol, and a mixture of $10 \%$ water and $90 \%$ glycerol by weight (see Table I). For each test, ten measurements of the jet length are taken, and the standard deviation from the mean value in all cases is less than $10 \%$. Sodium iodide is added to some of the liquids to provide a broad range of conductivity from 0.1 to $10 \mu \mathrm{S} / \mathrm{cm}$. Conductivity higher than $10 \mu \mathrm{S} / \mathrm{cm}$ is not considered in this study as at higher conductivity the jet tends to have nonaxisymmetric breakup.

The experimental setup consists of a tapered metal nozzle of outer diameter at the tip $d_{n}$ ranging between 30 
TABLE I. Physical properties of the liquids used in the experiments.

\begin{tabular}{|c|c|c|c|c|}
\hline Liquid & $\begin{array}{c}\rho \\
\left(\mathrm{kg} / \mathrm{m}^{3}\right)\end{array}$ & $\begin{array}{c}\mu \\
(\mathrm{kg} / \mathrm{ms})\end{array}$ & $\begin{array}{c}\gamma \\
(\mathrm{mN} / \mathrm{m})\end{array}$ & $\begin{array}{c}K \\
(\mu \mathrm{S} / \mathrm{cm})\end{array}$ \\
\hline 10 -wt $\%$ water and $90-w t \%$ glycerol & 1225 & 0.18 & 57 & 4.8 \\
\hline Glycerol $0.3 \mu \mathrm{S} / \mathrm{cm}$ & 1262 & 0.976 & 56.6 & 0.33 \\
\hline Ethylene glycol $0.78 \mu \mathrm{S} / \mathrm{cm}$ & 1106 & 0.019 & 19.3 & 0.78 \\
\hline Ethylene glycol $4 \mu \mathrm{S} / \mathrm{cm}$ & 1106 & 0.019 & 19.3 & 4 \\
\hline Ethylene glycol $10 \mu \mathrm{S} / \mathrm{cm}$ & 1106 & 0.019 & 19.3 & 10 \\
\hline Triethylene glycol & 1124 & 0.049 & 40 & 0.32 \\
\hline Dipropylene glycol & 1023 & 0.075 & 35.5 & 2 \\
\hline Propylene carbonate & 1200 & 0.0025 & 40.9 & 10 \\
\hline Propanol $0.1 \mu \mathrm{S} / \mathrm{cm}$ & 782 & 0.0024 & 19.6 & 0.11 \\
\hline Propanol $2 \mu \mathrm{S} / \mathrm{cm}$ & 782 & 0.0024 & 19.6 & 2.1 \\
\hline 1-Methoxy-2-propanol & 916 & 0.0018 & 27.7 & 0.2 \\
\hline Ethanol & 787 & 0.001 & 22 & 10 \\
\hline
\end{tabular}

and $250 \mu \mathrm{m}$. In all cases the fluid meniscus wetted the outer edge of this nozzle. This nozzle is connected to highvoltage power supply (Fug HCP 14-6500), which is able to supply a voltage difference of up to $10 \mathrm{kV}$ between the nozzle and a ground plane. A thin metal plate is used as the ground electrode. This plate has a 2-mm hole at the center to avoid flow accumulation on the electrode surface. The separation between the nozzle and the plate is set to be $3 \mathrm{~mm}$, this separation thus being much larger than the jet diameter. In view of this, the assumption of having the ground electrode at infinity in our analysis is justified. Images of the jet are captured with a CCD camera (uEye UI-2230-C, IDS) with a microscopic lens (Optem Zoom 125) and a flash lamp (High-Speed Photo-Systeme) with an illumination time of $20 \mathrm{~ns}$. The magnification of the microscopic lens can be adjusted to give a resolution ranging from one to four pixels per micrometer. A waveform generator (HP 33120A, Hewlett-Packard) and a delay-pulse generator (DG535, Stanford Research Systems) are used to synchronize the triggering pulses of the camera with the flash lamp. A diffuser is placed between the light source and the nozzle to ensure uniform lighting. The images are processed with image-analysis software (ImageJ) to measure the jet intact length.

The working fluid is fed into the nozzle by use of either a syringe pump (Harvard Apparatus PHD4400) or a height difference of the liquid level between the nozzle tip and a reservoir, with a flow rate $Q$ between 0.0006 and 0.1 $\mathrm{ml} / \mathrm{min}$. The flow rate is measured with a nanoflow sensor (model N-565, Upchurch Scientific). Fig. 1 shows the breakup length of the jet as a function of the flow rate for individual liquids. As one may expect, increase of the flow rate and/or the viscosity leads to a longer jet. Also, for the same solvent at different conductivity in Fig. 1, it may be seen that increased conductivity delays the breakup. This effect can be elucidated in terms of an increase of the electric field strength on the jet, which is known to stabilize conducting jets [17]. Also, the increase of conductivity yields a reduction of the jet diameter and, therefore, an increase of the Ohnesorge number. This increase would stabilize the jet too. On the other hand, we find that the voltage difference has no direct influence on the jet length itself. This can be explained simply in terms of insensitivity of both the field near the apex of the cone and that on the jet to the geometric conditions of the electrodes that create the field [18]. However, from our observations it is evident that increasing the voltage does lead to an increased cone angle, and hence it is the cone length in the axial direction that declines (see Fig 2). In addition, from our data it appears that the diameter of the nozzle does not have any influence on the jet breakup length. This is because the nozzle diameter is much greater than the jet diameter, and hence the jet breakup itself is a local mechanism. These observations are consistent with the scaling proposed in Eq. 8, where the breakup length is a function only of the flow rate and the liquid properties.

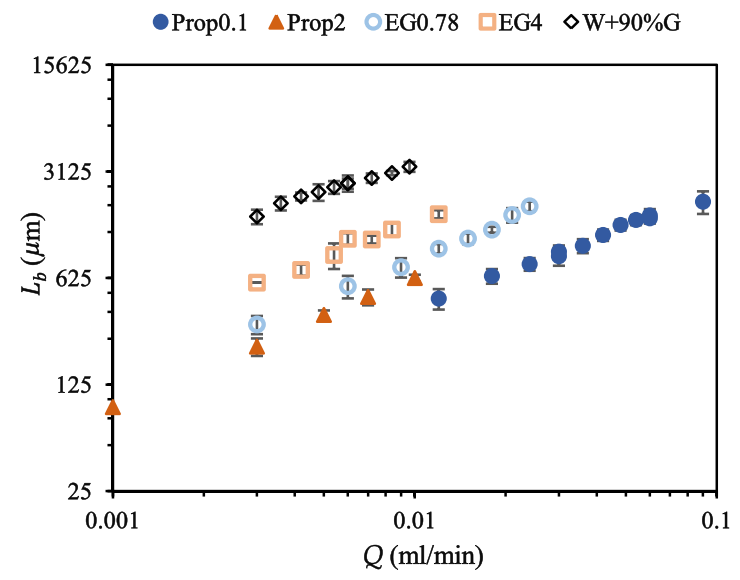

FIG. 1. Jet length as a function of the flow rate for liquids with different viscosities and conductivities. The nozzle diameter is $250 \mu \mathrm{m}$. EG0.78, ethylene glycol $0.78 \mu \mathrm{S} / \mathrm{cm}$; EG4, ethylene glycol $4 \mu \mathrm{S} / \mathrm{cm}$; Prop0.1, propanol $0.1 \mu \mathrm{S} / \mathrm{cm}$; Prop2, propanol $2 \mu \mathrm{S} / \mathrm{cm} ; \mathrm{W}+90 \% \mathrm{G}, 10$-wt $\%$ water and $90-\mathrm{wt} \%$ glycerol. 
(a)

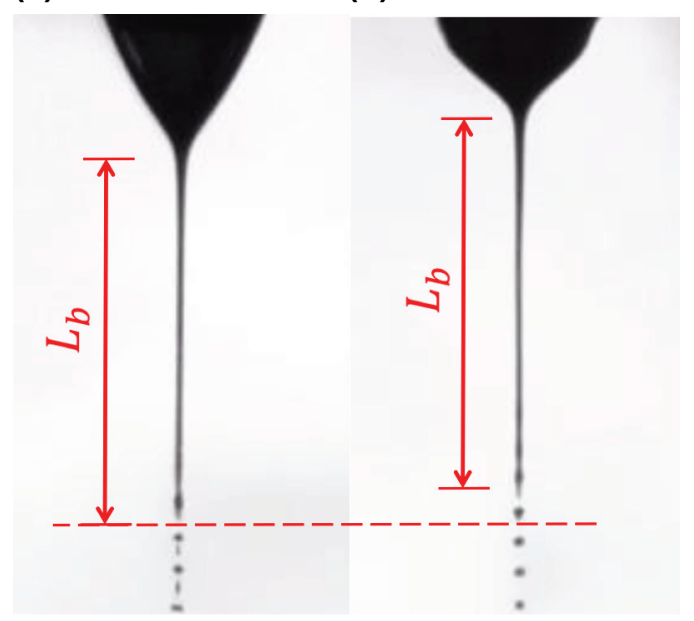

FIG. 2. Jet breakup of 1-methoxy-2-propanol at $Q=0.03 \mathrm{ml} /$ min and (a) $2500 \mathrm{~V}$ and (b) $3000 \mathrm{~V}$.

Figure 3 shows the measured breakup length normalized by $d_{n}$ for a total of 190 experiments against the theoretical model in Eq. (8). Excellent collapse for most of the data (open symbols) occurs when the constant $k_{v}$ has a value of 7 , with a maximum standard deviation of $16.8 \%$, for a data range that spans almost 3 orders of magnitude;

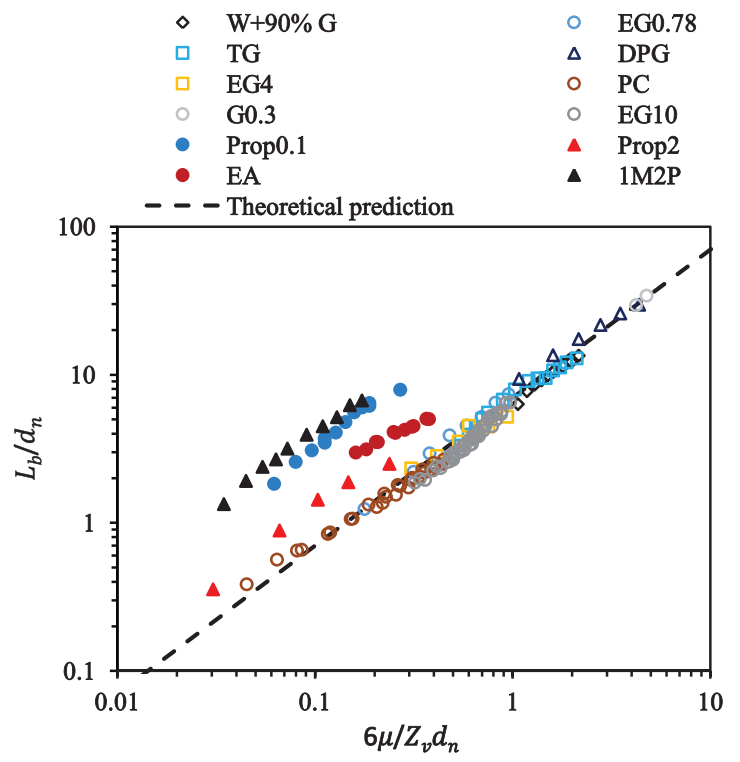

FIG. 3. Dimensionless breakup length $L_{b} / d_{n}$ as a function of the scaling variable $6 \mu / Z_{v} d_{n}$. Open symbols represent the data for $\operatorname{Re}<5$ and solid symbols represent the data for $\operatorname{Re}>$ 5. DPG, dipropylene glycol; EA, ethanol; EG0.78, ethylene glycol $0.78 \mu \mathrm{S} / \mathrm{cm}$; EG4, ethylene glycol $4 \mu \mathrm{S} / \mathrm{cm}$; EG10, ethylene glycol $10 \mu \mathrm{S} / \mathrm{cm} ; \mathrm{G}$, glycerol $0.3 \mu \mathrm{S} / \mathrm{cm}$; 1M2P, 1-methoxy-2-propanol; PC, propylene carbonate; Prop0.1, propanol $0.1 \mu \mathrm{S} / \mathrm{cm}$; Prop2, propanol $2 \mu \mathrm{S} / \mathrm{cm}$; TG, triethylene glycol; W+90\% G, 10-wt \% water and $90-\mathrm{wt} \%$ glycerol. this provides strong support for the proposed scaling law. The solid symbols, which diverge from the theoretical prediction, have electric Reynolds number $\operatorname{Re}>5$. The electric Reynolds number is defined here as $\mathrm{Re}=$ $\left[\left(\rho^{5} Q^{3} \gamma K\right) /\left(\mu \varepsilon_{0}\right)\right]^{1 / 6}$. This suggests that the scaling is limited to a low Reynolds number, which is consistent with our approach because the growth rate used in this analysis is valid only when viscosity is dominant.

\section{SCALING OF THE JET LENGTH IN THE INVISCID LIMIT}

To predict the breakup length for inviscid jets, one needs to estimate the growth rate when viscous effects are negligible in comparison with the inertia of the jet. If we replace the scale factors for time and velocity with $t_{c}=\left(\rho R^{3} / \gamma\right)^{1 / 2}$ and $v_{c}=(\rho R / \gamma)^{-1 / 2}$, respectively, in the inviscid limit, then Eq. (2) can be rewritten as

$$
\frac{\partial}{\partial t} \mathbf{v}=-\nabla p+\mathrm{Oh} \nabla^{2} \mathbf{v}
$$

Following Saville's approach, the general equation for the growth rate [5] is

$$
\frac{\hat{\omega} I_{m}(\xi)}{\xi I_{m}^{\prime}(\xi)}=1-m^{2}-\xi^{2}-\frac{R \varepsilon_{0} E_{0}^{2}}{4 \pi \gamma}\left[1+\xi \frac{K_{m}^{\prime}(\xi)}{K_{m}(\xi)}\right]
$$

where $\hat{\omega}$ is the growth rate in nondimensional form, $I_{m}$ and $K_{m}$ are the modified Bessel functions, $m$ is the azimuthal wavenumber, and $\xi$ is the axial wavenumber. If viscosity effect is negligible in comparison with the effect of inertia, $\hat{\omega}$ can be defined as $\omega\left(\rho R^{3} / \gamma\right)^{1 / 2}$. For axisymmetric motion, the general equation reduces to

$$
\frac{\omega \rho R^{3}}{\gamma}=\frac{1-\xi^{2}-\left(R \varepsilon_{0} E_{0}^{2} / 4 \pi \gamma\right)\left\{1+\xi\left[K_{0}^{\prime}(\xi) / K_{0}(\xi)\right]\right\}}{2\left\{\left[\xi I_{0}(\xi) / I_{1}(\xi)\right]^{2}-\xi^{2}-1\right\}} .
$$

For long wavelength $(\xi \rightarrow 0)$, where the most-unstable axisymmetric breakup is observed, the growth rate in the inviscid limit can be written as

$$
\omega^{2} \sim \frac{\gamma}{6 \rho R^{3}}\left(1-\frac{R \varepsilon_{0} E_{0}^{2}}{4 \pi \gamma}\right)
$$

This is similar to Rayleigh's expression for an inviscid jet produced from a charged liquid sphere [19]. On the basis of Eq. (13), it is easy to estimate the breakup length in the inviscid limit as

$$
L_{b}=k_{i} \frac{6}{Z_{i}}
$$




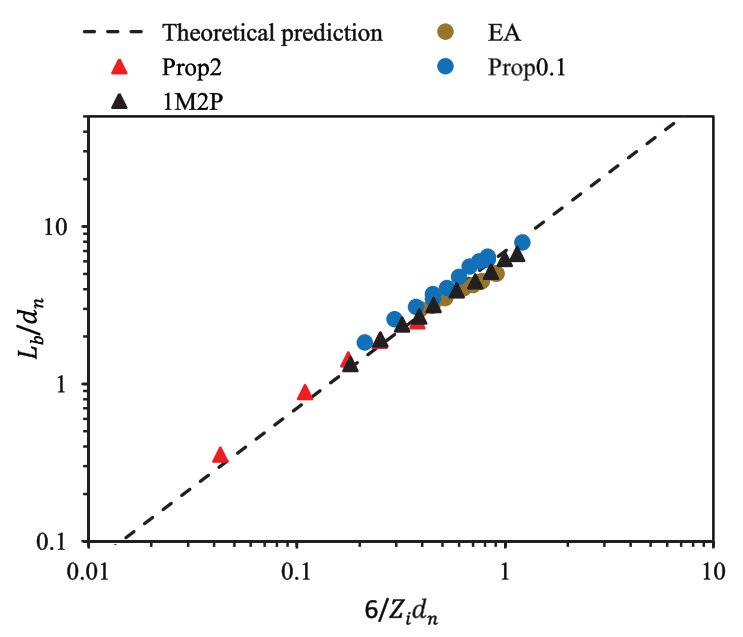

FIG. 4. Dimensionless breakup length $L_{b} / d_{n}$ as a function of the scaling variable $6 / Z_{i} d_{n}$. EA, ethanol; 1M2P, 1-methoxy2-propanol; Prop0.1, propanol $0.1 \mu \mathrm{S} / \mathrm{cm}$; Prop2, propanol $2 \mu \mathrm{S} / \mathrm{cm}$.

where

$$
Z_{i}=\left[\left(\frac{\gamma^{5} \varepsilon_{0}}{\rho^{5} Q^{9} K}\right)^{1 / 6}-\frac{1}{4 \pi}\left(\frac{\gamma K}{\rho Q^{3} \varepsilon_{0}}\right)^{1 / 3}\right]^{1 / 2} .
$$

Comparison of our experimental data in the inviscid limit with the new scaling in Eq. (14) this shows excellent agreement with a fitting factor $k_{i}$ of 7 (see Fig. 4). The maximum standard deviation, with data over 2 orders of magnitude, is $15 \%$. It should be emphasized that the collapse in Fig. 3 happens only for the data for which $\operatorname{Re}>5$. This observation provides cogent evidence of the credibility of the two models, wherein each model is valid only within the limits proposed in the analysis.
TABLE II. Physical properties of the inks used in the printing.

\begin{tabular}{lcccc}
\hline \hline Ink & $\begin{array}{c}\rho \\
\left(\mathrm{kg} / \mathrm{m}^{3}\right)\end{array}$ & $\begin{array}{c}\mu \\
(\mathrm{kg} / \mathrm{m} \mathrm{s})\end{array}$ & $\begin{array}{c}\gamma \\
(\mathrm{mN} / \mathrm{m})\end{array}$ & $\begin{array}{c}K \\
(\mu \mathrm{S} / \mathrm{cm})\end{array}$ \\
\hline $\begin{array}{c}55 \text {-wt\% silver } \\
\text { nanoparticles }\end{array}$ & 5700 & 1 & 40 & 0.01 \\
$\begin{array}{c}\text { 50-wt \% copper } \\
\text { nanoparticles }\end{array}$ & 4980 & 0.8 & 35 & 0.05 \\
\hline \hline
\end{tabular}

In the following section, we illustrate the importance of our models for improving the electrostatic-inkjet-printing technology.

\section{PRINTING METAL GRIDS WITH HIGH QUALITY AND HIGH RESOLUTION}

Direct printing of metal grids with good optical and electrical performance shows great potential to fabricate transparent conductive electrodes, especially in the case when high resolution of metal grids is preferred to provide invisibility and when flexibility of electrodes is needed for flexible devices.

In this section, the objective is to print uniform grid lines from nanoparticle inks with the minimum possible width to enhance the resolution. We use silver and copper nanoparticle inks with the physical properties given in Table II. The printing system consists of a glass nozzle with a tip diameter of $50 \mu \mathrm{m}$. A metal needle is inserted inside the glass-tube nozzle to work as an electrode. This electrode is connected to a high-voltage power supply. The glass nozzle is fixed to the print-head body, which has two inlets: one inlet is for the electrode; the other inlet is connected to a tube with a reservoir at its end to introduce the ink. A high-speed $X-Y$ stage (AeroTech PRO $165 \mathrm{LM}$ ) is used to move a glass substrate placed on a metal plate horizontally

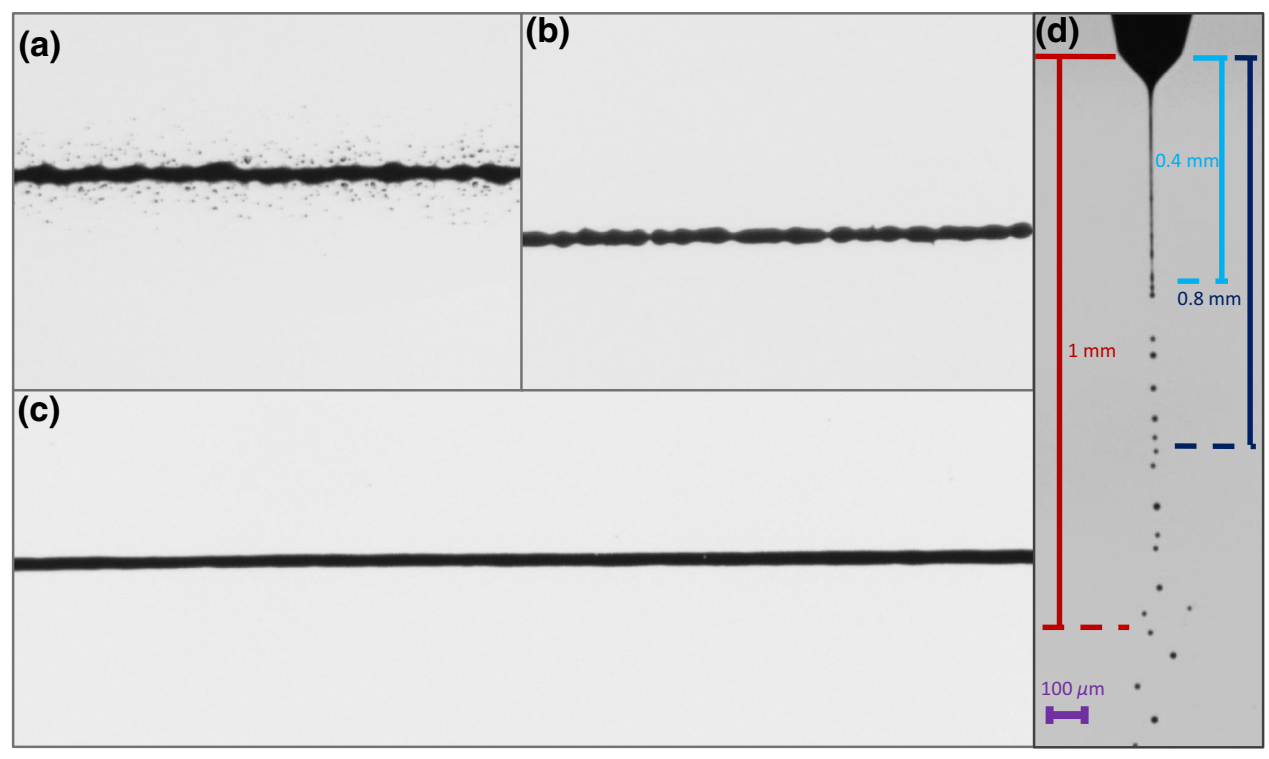

FIG. 5. Copper lines printed at a printing distance of (a) $1 \mathrm{~mm}$ (b) $0.8 \mathrm{~mm}$, and (c) $0.4 \mathrm{~mm}$. The jet breakup during the printing is shown in (d). 


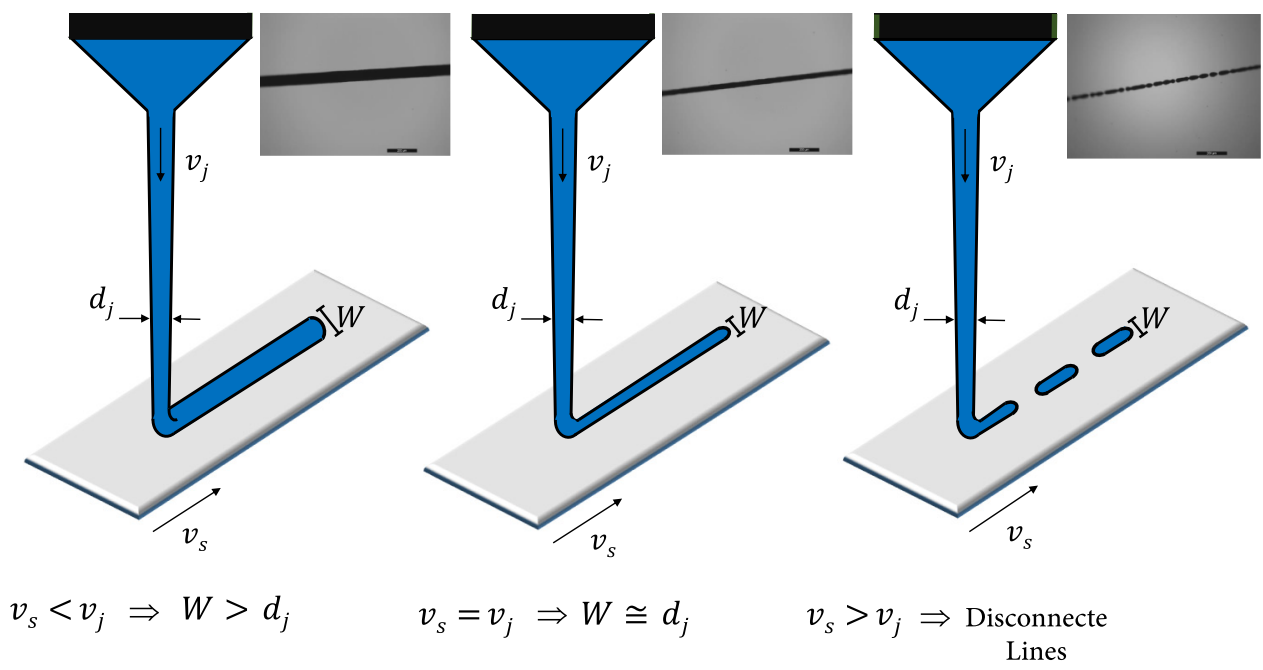

FIG. 6. Influence of the stage velocity and the jet velocity on the line width.

in the $X-Y$ plane during the printing. This plate acts as the ground electrode. Initially we set the printing distance between the nozzle and the substrate to be 0.8 and $1 \mathrm{~mm}$.

Figures 5(a) and 5(b) show the printed lines in both cases. It can be seen that the lines are not uniform, because the printing distance is greater than the jet intact length. In the case of the longest print distance $(1 \mathrm{~mm})$, there is enough distance for the satellite droplets that separate from the postbreakup jet to measurably divert from the axis and produce the scattered shape of the lines shown in Fig. 5(a). This is confirmed by our imaging the jet during printing [see Fig. 5(d)]. In Fig. 5(b), where the printing distance is $0.8 \mathrm{~mm}$, the scattering has disappeared because now although the droplets still divert, the deviation following the breakup cannot be resolved from the line width printed, but the line is still nonuniform. This is because the line is formed by the coalescence of subsequent droplets. In that case the uniformity of the line is limited to a narrow stable region [20]. One simple method then to avoid the nonuniformity problem is to set the printing distance less than the jet length. In that way, the jet will be deposited directly on the substrate, forming a continuous uniform line, as can be seen in Fig. 5(c).

The printed line width is limited at minimum to the jet diameter; when the stage velocity is equal to the jet velocity, the line width will be close to the jet diameter. If the stage velocity is less than the jet velocity, the line width will be greater than the jet diameter, and if the stage velocity is greater than the jet velocity, the line will be disconnected (see Fig. 6). The key point then to have a thinner line is to decrease the jet diameter and keep the stage velocity $v_{s}$ equal to the jet velocity $v_{j}$.

To do so, we can simply reduce the flow rate. The problem is that this would shorten the jet below the printing distance, so we will need to decrease the printing distance to avoid having nonuniform lines. From this we conclude that the resolution of the printed lines is limited to the minimum printing distance that we can set in the system.
The previous analysis gives a rough estimation of the line width. For a more-accurate prediction, the interaction between the liquid and the substrate's surface has to be considered.

The width $W$ is a function of the cross-section area of the printed line $A_{l}$ and the contact angle $\theta$. The area can be calculated as a segment of circle as follows:

$$
A_{l}=\frac{r^{2}}{2}(2 \theta-\sin 2 \theta)
$$

where $r=W / 2 \sin \theta$.

If we assume that the stage velocity is set to be equal to the jet velocity as discussed earlier, then the line crosssection area should be equal to the cross-section area of the jet $A_{j}=\pi d_{j}^{2} / 4$. Finally, the line width can be expressed in terms of the jet diameter and the contact angle as follows:

$$
W=d_{j} \sin \theta \sqrt{\frac{2 \pi}{2 \theta-\sin 2 \theta}} .
$$

To print a uniform silver grid with lines $10 \mu \mathrm{m}$ in width, we need to calculate the corresponding jet diameter from Eq. (17). The measured contact angle between the silver ink and the glass substrate is $14^{\circ}$; as a result, the jet diameter should be $2.2 \mu \mathrm{m}$. The flow rate that gives

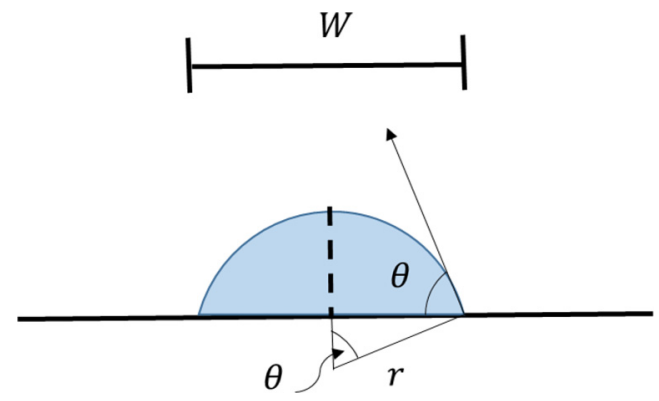

FIG. 7. Cross-section area of the printed line. 
(a)

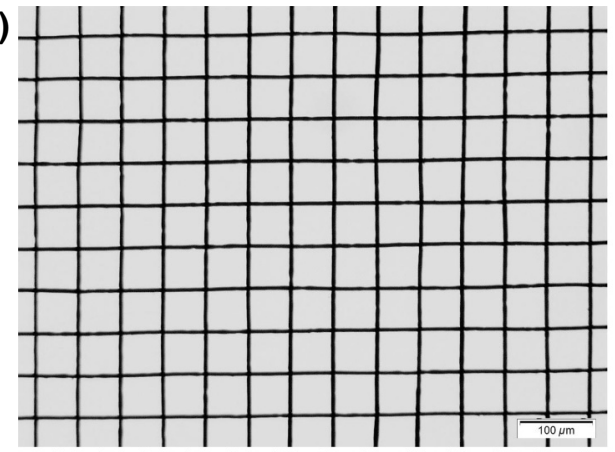

(b)

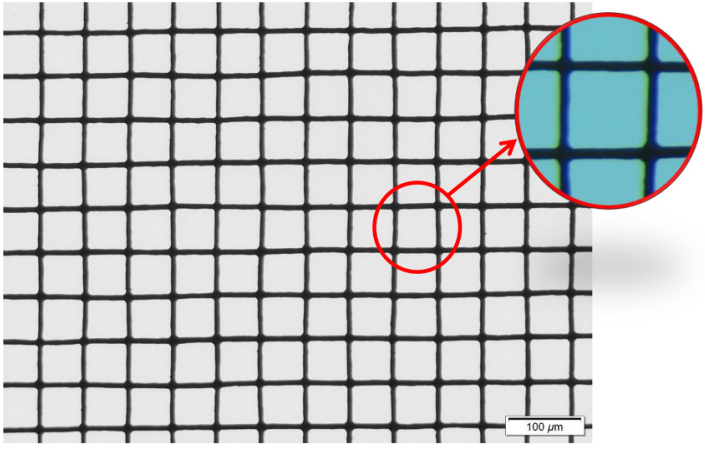

FIG. 8. Metal grids printed from (a) silver nanoparticle ink and (b) copper nanoparticle ink.

a jet $2.2 \mu \mathrm{m}$ in diameter should be set in the printing system. This flow rate can be estimated from Eq. (6), $\left(R^{6} \gamma K / \rho \varepsilon_{0}\right)^{1 / 3}=0.05 \mu \mathrm{l} / \mathrm{min}$. The stage velocity $v_{s}=$ $v_{j}=4 Q / \pi d_{j}^{2}=294 \mathrm{~mm} / \mathrm{s}$. Finally, to ensure that the lines will be uniform, the printing distance should be set less than the jet intact length, which can be calculated from Eq. (8), $\left(6 k_{v} \mu / Z_{v}\right)=709 \mu \mathrm{m}$, as $\operatorname{Re}<O(1)$ in this case. Following the previous printing conditions, a very thin uniform silver grid lines are successfully printed as illustrated in Fig. 8(a). The same approach is adopted to print a copper grid as shown in Fig. 8(b). The line width in both cases is around $10 \mu \mathrm{m}$, as we anticipated.

\section{CONCLUSION}

In the present work, we derive scaling laws of the intact length of an electrified jet in two different regimes. The scaling laws are validated with experimental data obtained with a wide range of governing parameters. The jet intact length is independent of the voltage applied to the electrospray. The voltage has an effect only on the cone angle and hence the cone axial length. Further, the jet length is not affected by the nozzle size because $d_{n} \gg R$. On the basis of competition between inertia and the viscous force, the regime in which the jet breaks up is identified. If the viscous force is dominant compared with inertia, being $\operatorname{Re} \lesssim O(1)$, the breakup length $L_{b} \sim 6 \mu / Z_{v}$, while for Re $>O(1)$, the scaling is $L_{b} \sim 6 / Z_{i}$. The experimental results reveal a threshold validity limit between the two scaling laws at $\mathrm{Re} \simeq 5$.
These results have an impact on a wide range of application areas, including chemical engineering, the textile industry, and inkjet printing. We show how these scaling laws are of great importance to enhance the quality and resolution of the printed lines in electrostatic-inkjet-printing applications as an example of the practical impact of these models. On the basis of our analysis, we finally present an accurate prediction of the line width as a function of the jet diameter and the contact angle.

\section{ACKNOWLEDGMENTS}

This work was supported by the Engineering and Physical Sciences Research Council (United Kingdom) under Grant No. EP/N509917/1 and the European Union's Horizon 2020 research and innovation program under Grant No. 646296.

[1] Lord Rayleigh, On the instability of jets, Proc. London Math. Soc. s1-10, 4 (1878).

[2] A. Javadi, J. Eggers, D. Bonn, M. Habibi, and N. M. Ribe, Delayed Capillary Breakup of Falling Viscous Jets, Phys. Rev. Lett. 110, 144501 (2013).

[3] G. Taylor, Electrically driven jets, Proc. R. Soc. London A 313, 453 (1969).

[4] A. B. Basset, Waves and jets in a viscous liquid, Am. J. Math. 16, 93 (1894).

[5] D. A. Saville, Stability of electrically charged viscous cylinders, Phys. Fluids 14, 1095 (1971).

[6] D. T. Conroy, K. Matar, R. V. Craster, and D. T. Papageorgiou, Breakup of an electrified viscous thread with charged surfactants, Phys. Fluids 23, 022103 (2011).

[7] Robert T. Collins, Michael T. Harris, and Osman A. Basaran, Breakup of electrified jets, J. Fluid Mech. 588, 75 (2007).

[8] J. Shercliff, Field-coupled surface waves, J. Fluid Mech. 21, 764 (1965).

[9] A. L. Huebner, and H. N. Chu, Instability and breakup of charged liquid jets, J. Fluid Mech. 49, 361 (1971).

[10] A. J. Mestel, Electrohydrodynamic stability of a slightly viscous jet, J. Fluid Mech. 274, 93 (1994).

[11] A. J. Mestel, Electrohydrodynamic stability of a highly viscous jet, J. Fluid Mech. 312, 311 (1996).

[12] M. Cloupeau, and B. Prunet-Forch, Electrostatic spraying of liquids in cone-jet mode, J. Electrostat. 22, 135 (1989).

[13] J. M. López-Herrera, P. Riesco-Chueca, and A. M. GanánCálvo, Linear stability analysis of axisymmetric perturbations in imperfectly conducting liquid jets, Phys. Fluids 17, 034106 (2005).

[14] A. M. Ganán-Calvo, J. M. López-Herrera, N. RebolloMunoz, and J. M. Montanero, The onset of electrospray: The universal scaling laws of the first ejection, Sci. Rep. 6, 32357 (2016).

[15] A. M. Ganán-Calvo, On the general scaling theory for electrospraying, J. Fluid Mech. 507, 203 (2004). 
[16] Alfonso M. Ganán-Calvo, José M. López-Herrera, Miguel A. Herrada, Antonio Ramos, and José M. Montanero, Review on the physics of electrospray: From electrokinetics to the operating conditions of single and coaxial Taylor cone-jets, and ac electrospray, J. Aerosol. Sci. 125, 32 (2018).

[17] F. Li, X. Y. Yin, and X. Z. Yin, Linear instability of a coflowing jet under an axial electric field, Phys. Rev. E 74 (2006).
[18] J. Fernández de la Mora, and I. G. Loscertales, The current emitted by highly conducting Taylor cones, J. Fluid Mech. 260, 155 (1994).

[19] Lord Rayleigh, XX. On the equilibrium of liquid conducting masses charged with electricity, Philos. Mag. 14, 184 (1882).

[20] Jonathan Stringer, and Brian Derby, Formation and stability of lines produced by inkjet printing, Langmuir 26, 10365 (2010). 